\title{
Optimal control to limit the spread of COVID-19 in Italy
}

\author{
Mohamed Abdelaziz Zaitri ${ }^{1,2}$, Mohand Ouamer Bibi ${ }^{1}$, Delfim F. M. Torres ${ }^{2, *}$ \\ ${ }^{1}$ Dept. of Operational Research, Research Unit LaMOS (Modeling and \\ Optimization of Systems), University of Bejaia, 06000 Bejaia, Algeria \\ ${ }^{2}$ Dept. of Mathematics, Center for Research and Development in Mathematics \\ and Applications (CIDMA), University of Aveiro, 3810-193 Aveiro, Portugal \\ *Corresponding author:delfim@ua.pt
}

\begin{abstract}
We apply optimal control theory to a generalized SEIR-type model. The proposed system has three controls, representing social distancing, preventive means, and treatment measures to combat the spread of the COVID-19 pandemic. We analyze such optimal control problem with respect to real data transmission in Italy. Our results show the appropriateness of the model, in particular with respect to the number of quarantined/hospitalized (confirmed and infected) and recovered individuals. Considering the Pontryagin controls, we show how in a perfect world one could have drastically diminish the number of susceptible, exposed, infected, quarantined/hospitalized, and death individuals, by increasing the population of insusceptible/protected.
\end{abstract}

Keywords: Analysis of the spread of COVID-19; Control system; Mathematical modeling; Optimal control; Pontryagin extremals.

\section{Introduction}

A severe outbreak of respiratory illness started in Wuhan, a city of eleven million people in central China, in December 2019. The causative agent was the novel severe acute respiratory syndrome coronavirus 2 (SARS-CoV-2), which was identified and isolated from a single patient in early January 2020 and subsequently verified in sixteen additional patients. The virus is believed to have a zoonotic origin. In particular, the Huanan Seafood Market, a live animal and seafood wholesale market in Wuhan, was regarded as a primary source of this epidemic, as it is found that $55 \%$ of the first four hundred twentyfive confirmed cases were linked to the marketplace. Meanwhile, recent comparisons of the genetic sequences of this virus and bat coronaviruses show a 96\% similarity (Lu et al., 2020).

Multiple mathematical models were already presented to predict the dynamics of this pandemic at a regional and global level, and some of these models were implemented, following different methods, to evaluate a strategy for preventive measures: in (Bagal et al., 2020), the classical susceptible-infectedrecovered (SIR) modeling approach (Kermack \& McKendrick, 1927) was employed to study the parameters of this model for India while considering different governmental lockdown measures; in (Lauer et al., 2020), the length of the incubation period of COVID-19 is estimated using confirmed COVID-19 cases reported between January 4 and February 24, 2020, from fifty provinces, regions, and counties from China; in (Lin et al., 2020) a model of the outbreak in Wuhan, with individual reaction and governmental action (holiday extension, city lockdown, hospitalization and quarantine) is analyzed in the light of the 1918 influenza pandemic in London; in (Moussaoui \& Auger, 2020), susceptible-exposedinfectious-recovered (SEIR) modeling is considered to forecast the COVID-19 outbreak in Algeria by 
using real data from March 1 to April 10, 2020; in (Zhao et al., 2020), a modified SEIR model is considered under three intervention scenarios (suppression, mitigation, mildness) and simulated to predict and investigate the realities in several African countries: South Africa, Egypt, Algeria, Nigeria, Senegal and Kenya. The list of such studies is long: see, e.g., (Lemos-Paião et al., 2020) for a new compartmental epidemiological model for COVID-19 with a case study of Portugal; (Ndaïrou et al., 2021) for a fractional (non-integer order) model applied to COVID-19 in Galicia, Spain and Portugal; (Zine et al., 2020) for a stochastic time-delayed COVID-19 model with application to the Moroccan deconfinement strategy; etc.

In (Peng et al., 2020), a mathematical system, generalizing the SEIR model, is presented to analyze the COVID-19 epidemic based on a dynamic mechanism that incorporates the intrinsic impact of hidden latent and infectious cases on the entire process of the virus transmission. The authors of (Peng et al., 2020) validate their model by analyzing data correlation on public data of the National Health Commission of China from January 20 to February 9, 2020, and produce reliable estimates and predictions, revealing key parameters of the COVID-19 epidemic. Here, we modify the model analyzed in (Peng et al., 2020) in order to consider optimal control problems. More precisely, we introduce three control variables and combine them with the main parameters of the model of (Peng et al., 2020). Secondly, we analyze a concrete optimal control problem, solving it analytically through the celebrated Pontryagin minimum principle (Pontryagin et al., 1962). Moreover, we perform numerical simulations of the spread of COVID-19 in Italy from September 1 to November 30, 2020. The model of (Peng et al., 2020) has shown to be a good model to describe the reality of China. Its weakness is that it just tries to describe a reality but without controlling it. Our main purpose and contribution here is to include control measures that allow us to interfere with reality. Moreover, we want to illustrate the validity of the model in a different context. For this reason, we have considered real data of COVID-19 from Italy instead of China.

The paper is organized as follows. In Section 2, we recall the generalized SEIR model of (Peng et al., 2020). Our original results begin with Section 3, where we introduce a generalized SEIR control system. An optimal control problem is posed and solved analytically in Section 4. Then, in Section 5, we estimate the parameters of the model using real data of COVID-19 from Italy, and we illustrate the usefulness of the proposed optimal control problem through numerical simulations. Our results show that the generalized SEIR model of (Peng et al., 2020), originally considered for China, is also effective with respect to Italy, being able to model well available real data, while our optimal control approach shows clearly the positive and crucial effects of social distancing, preventive means, and treatment in the combat of COVID-19. We end with Section 6 of conclusions.

\section{A generalized SEIR-type model}

The classical SEIR model consists of four compartments: susceptible individuals $S(t)$, exposed individuals $E(t)$, infected individuals $I(t)$, recovered individuals $R(t)$. This SEIR model is too simplistic to describe COVID-19 epidemic and new classes need to be included, e.g., Deaths and Quarantined individuals, in order to describe the reality. A generalized SEIR-type model for COVID-19 is proposed by Peng et al. (Peng et al., 2020), being expressed by a seven-dimensional dynamical system as follows:

$$
\left\{\begin{array}{l}
\dot{S}(t)=-\frac{\beta S(t) I(t)}{N}-\alpha S(t), \\
\dot{E}(t)=\frac{\beta S(t) I(t)}{N}-\gamma E(t), \\
\dot{I}(t)=\gamma E(t)-\delta I(t), \\
\dot{Q}(t)=\delta I(t)-\lambda(t) Q(t)-\kappa(t) Q(t), \\
\dot{R}(t)=\lambda(t) Q(t), \\
\dot{D}(t)=\kappa(t) Q(t), \\
\dot{P}(t)=\alpha S(t),
\end{array}\right.
$$


subject to fixed initial conditions

$$
S(0)=S_{0}, E(0)=E_{0}, I(0)=I_{0}, Q(0)=Q_{0}, R(0)=R_{0}, D(0)=D_{0}, P(0)=P_{0} .
$$

Here, the population is divided into susceptible individuals $S(t)$, exposed individuals $E(t)$, infected individuals $I(t)$, quarantined/hospitalized individuals (confirmed and infected) $Q(t)$, recovered individuals $R(t)$, death individuals $D(t)$, and insusceptible individuals (protected population) $P(t)$. It follows from (1) that

$$
\dot{S}(t)+\dot{E}(t)+\dot{I}(t)+\dot{Q}(t)+\dot{R}(t)+\dot{D}(t)+\dot{P}(t)=0,
$$

so that

$$
S(t)+E(t)+I(t)+Q(t)+R(t)+D(t)+P(t)
$$

is constant along time $t$. This constant will be denoted by $N$, being determined by the initial conditions (2):

$$
N:=S_{0}+E_{0}+I_{0}+Q_{0}+R_{0}+D_{0}+P_{0} .
$$

The constant parameters $\alpha, \beta, \gamma$ and $\delta$ represent, respectively, the protection rate, infection rate, inverse of the average latent time, and the rate at which infectious people enter in quarantine, and they have the dimension of time ${ }^{-1}\left(\right.$ day $\left.^{-1}\right)$. The recovery and mortality rates, respectively $\lambda$ and $\kappa$, are time-dependent analytical functions defined by

$$
\lambda(t):=\frac{\lambda_{1}}{1+e^{-\lambda_{2}\left(t-\lambda_{3}\right)}}
$$

and

$$
\kappa(t):=\frac{\kappa_{1}}{e^{\kappa_{2}\left(t-\kappa_{3}\right)}+e^{-\kappa_{2}\left(t-\kappa_{3}\right)}},
$$

where the parameters $\lambda_{1}, \lambda_{2}, \lambda_{3}, \kappa_{1}, \kappa_{2}$ and $\kappa_{3}$ are determined empirically from real data. Note that $\lambda_{1}$, $\lambda_{2}, \kappa_{1}$ and $\kappa_{2}$ have the dimension of time ${ }^{-1}\left(\right.$ day $\left.^{-1}\right)$, while $\lambda_{3}$ and $\kappa_{3}$ have the dimension of time (day).

Remark 1 The basic reproduction number is usually computed for autonomous systems, when the righthand side of the system does not depend explicitly on time $t$ (Barril et al., 2021; van den Driessche \& Watmough, 2002). Here, system (1) depends on (3) and, therefore, it is a non-autonomous system. In this case, we are not aware of a valid method to compute the basic reproduction number.

\section{Formulation of the Problem}

We introduce three time-dependent controls to model (1) of (Peng et al., 2020):

- control $u_{1}(t)$, representing the effect of social distancing;

- control $u_{2}(t)$, representing the effect of preventive means;

- control $u_{3}(t)$, representing the effect of treatment.

Mathematically, we have the control system

$$
\left\{\begin{array}{l}
\dot{S}(t)=-\frac{\beta u_{1}(t) S(t) I(t)}{N}-\left(\alpha+u_{2}(t)\right) S(t), \\
\dot{E}(t)=\frac{\beta u_{1}(t) S(t) I(t)}{N}-\gamma E(t) \\
\dot{I}(t)=\gamma E(t)-\delta I(t) \\
\dot{Q}(t)=\delta I(t)-\left(\lambda(t)+u_{3}(t)\right) Q(t)-\kappa(t) Q(t), \\
\dot{R}(t)=\left(\lambda(t)+u_{3}(t)\right) Q(t) \\
\dot{D}(t)=\kappa(t) Q(t) \\
\dot{P}(t)=\left(\alpha+u_{2}(t)\right) S(t)
\end{array}\right.
$$




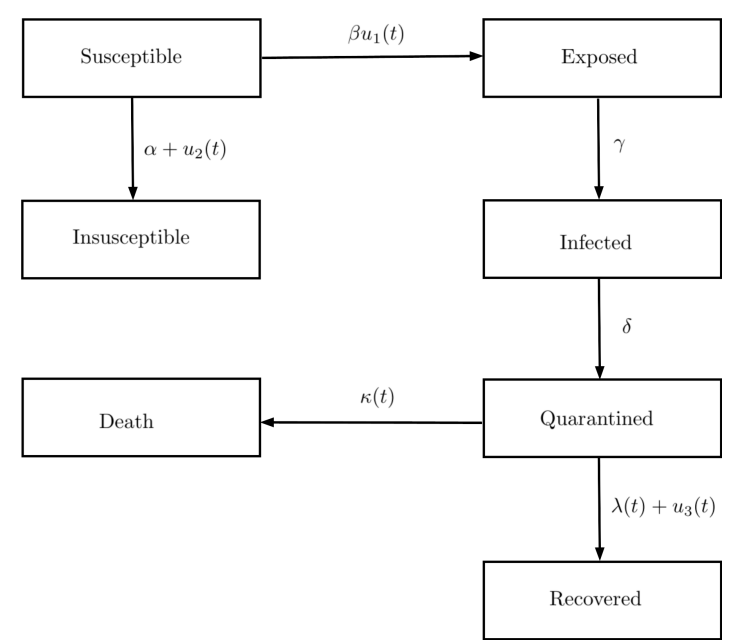

Fig. 1. Schematic diagram of the generalized SEIR-type control system (5).

subject to initial conditions (2). We call (5) the generalized SEIR-type control model. A schematic diagram of our control system is given in Figure 1.

\section{Optimal Control}

We consider the generalized SEIR control model (5) and formulate an optimal control problem to determine the strategy $u(t)=\left(u_{1}(t), u_{2}(t), u_{3}(t)\right)$, over a fixed interval of time $\left[0, t_{f}\right]$, that minimizes the cost functional

$$
J(u)=\int_{0}^{t_{f}}\left(w_{1} \frac{\beta u_{1} S(t) I(t)}{N}-w_{2} R(t)-w_{3} P(t)+v_{1} \frac{u_{1}^{2}}{2}+v_{2} \frac{u_{2}^{2}}{2}+v_{3} \frac{u_{3}^{2}}{2}\right) d t,
$$

where $t_{f}$ represents the final time of the period under study and the constants $w_{1}, w_{2}, w_{3}, v_{1}, v_{2}$ and $v_{3}$ represent the weights associated with the total number of new infections, the number of recovered individuals, the number of insusceptible individuals, and the costs associated with the controls $u_{1}, u_{2}$ and $u_{3}$, respectively. The controls $u$ are Lebesgue measurable and bounded:

$$
u(t) \in \Gamma:=\left\{\mu=\left(\mu_{1}, \mu_{2}, \mu_{3}\right) \in \mathbb{R}^{3}: u_{i \min } \leq \mu_{i} \leq u_{i \max }, i=1,2,3\right\} .
$$

The intervals $\left[u_{i \min }, u_{i \max }\right]$ also translate the fact that there are limitations to the effects of social distancing, the preventive means and the treatment rate. Let

$$
\begin{aligned}
x(t) & =\left(x_{1}(t), \ldots, x_{7}(t)\right) \\
& =(S(t), E(t), I(t), Q(t), R(t), D(t), P(t)) \in \mathbb{R}^{7} .
\end{aligned}
$$

The optimal control problem consists to find the optimal trajectory $\tilde{x}$ associated with the optimal control $\tilde{u} \in L^{1}, \tilde{u}(t) \in \Gamma$, satisfying the control system (5), the initial conditions

$$
x(0)=\left(S_{0}, E_{0}, I_{0}, Q_{0}, R_{0}, D_{0}, P_{0}\right)
$$

and giving minimum value to (6).

The existence of an optimal control $\tilde{u}$ and associated optimal trajectory $\tilde{x}$ comes from the convexity of the integrand of the cost functional (6) with respect to control $u$ and the Lipschitz property of the state system with respect to state variables $x$ (see (Cesari, 1983) for existence results of optimal solutions).

According to the Pontryagin Minimum Principle (Pontryagin et al., 1962), if $\tilde{u} \in L^{1}$ is optimal for problem (5)-(8) and fixed final time $t_{f}$, then there exists $\psi \in A C\left(\left[0, t_{f}\right] ; \mathbb{R}^{7}\right), \psi(t)=\left(\psi_{1}(t), \ldots, \psi_{7}(t)\right)$, 
called the adjoint vector, such that

$$
\left\{\begin{array}{l}
\dot{x}=\frac{\partial H}{\partial \psi}, \\
\dot{\psi}=-\frac{\partial H}{\partial x},
\end{array}\right.
$$

where the Hamiltonian $H$ is defined by

$$
H(x, u, \psi)=\frac{w_{1} u_{1} \beta x_{1} x_{3}}{N}-w_{2} x_{5}-w_{3} x_{7}+\sum_{i=1}^{3} v_{i} \frac{u_{i}^{2}}{2}+\psi^{T}\left(A x+\left(\sum_{i=1}^{2} b_{i} \Lambda_{i} x \Phi_{i}+f(x)^{T} \Phi_{3}\right) u\right)
$$

with

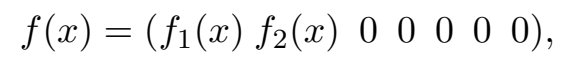

$$
\begin{aligned}
& f_{1}(x)=\frac{-\beta x_{1} x_{3}}{N}, \\
& f_{2}(x)=\frac{\beta x_{1} x_{3}}{N}, \\
& b_{1}=\left(\begin{array}{lllllll}
-1 & 0 & 0 & 0 & 0 & 0 & 0
\end{array}\right)^{T},
\end{aligned}
$$

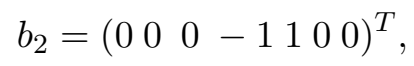

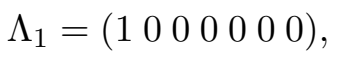

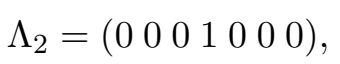

$$
\begin{aligned}
& \Phi_{1}=\left(\begin{array}{lll}
0 & 1 & 0
\end{array}\right), \\
& \Phi_{2}=\left(\begin{array}{lll}
0 & 0 & 1
\end{array}\right), \\
& \Phi_{3}=\left(\begin{array}{lll}
1 & 0 & 0
\end{array}\right), \\
& A=\left(\begin{array}{ccccccc}
-\alpha & 0 & 0 & 0 & 0 & 0 & 0 \\
0 & -\gamma & 0 & 0 & 0 & 0 & 0 \\
0 & \gamma & -\delta & 0 & 0 & 0 & 0 \\
0 & 0 & \delta & -\lambda(t)-\kappa(t) & 0 & 0 & 0 \\
0 & 0 & 0 & \lambda(t) & 0 & 0 & 0 \\
0 & 0 & 0 & \kappa(t) & 0 & 0 & 0 \\
\alpha & 0 & 0 & 0 & 0 & 0 & 0
\end{array}\right) .
\end{aligned}
$$

The minimality condition

$$
H(\tilde{x}(t), \tilde{u}(t), \tilde{\psi}(t))=\min _{u \in \Gamma} H(\tilde{x}(t), u, \tilde{\psi}(t))
$$

holds almost everywhere on $\left[0, t_{f}\right]$. Moreover, the transversality conditions

$$
\tilde{\psi}_{i}\left(t_{f}\right)=0, \quad i=1, \ldots, 7
$$

hold. Solving the minimality condition (9) on the interior of the set of admissible controls $\Gamma$ gives

$$
\tilde{u}(t)=\left(\frac{\beta \tilde{x}_{1}(t) \tilde{x}_{3}(t)\left(\tilde{\psi}_{1}(t)-\tilde{\psi}_{2}(t)-w_{1}\right)}{N v_{1}}, \frac{\tilde{x}_{1}(t)\left(\tilde{\psi}_{1}(t)-\tilde{\psi}_{7}(t)\right)}{v_{2}}, \frac{\tilde{x}_{4}(t)\left(\tilde{\psi}_{4}(t)-\tilde{\psi}_{5}(t)\right)}{v_{3}}\right),
$$


where the adjoint functions satisfy

$$
\left\{\begin{array}{l}
\dot{\tilde{\psi}}_{1}=-\frac{\tilde{u}_{1} \beta \tilde{x}_{3}}{N^{2}}\left(\tilde{x}_{2}+\tilde{x}_{3}+\tilde{x}_{4}+\tilde{x}_{5}+\tilde{x}_{6}+\tilde{x}_{7}\right)\left(w_{1}-\tilde{\psi}_{1}+\tilde{\psi}_{2}\right)+\left(\alpha+\tilde{u}_{2}\right)\left(\tilde{\psi}_{1}-\tilde{\psi}_{7}\right), \\
\dot{\tilde{\psi}}_{2}=\frac{\tilde{u}_{1} \beta \tilde{x}_{1} \tilde{x}_{3}\left(w_{1}-\tilde{\psi}_{1}+\tilde{\psi}_{2}\right)}{N^{2}}+\gamma\left(\tilde{\psi}_{2}-\tilde{\psi}_{3}\right), \\
\dot{\tilde{\psi}}_{3}=-\frac{\tilde{u}_{1} \beta \tilde{x}_{1}}{N^{2}}\left(\tilde{x}_{2}+\tilde{x}_{3}+\tilde{x}_{4}+\tilde{x}_{5}+\tilde{x}_{6}+\tilde{x}_{7}\right)\left(w_{1}-\tilde{\psi}_{1}+\tilde{\psi}_{2}\right)+\delta\left(\tilde{\psi}_{3}-\tilde{\psi}_{4}\right), \\
\dot{\tilde{\psi}}_{4}=\frac{\tilde{u}_{1} \beta \tilde{x}_{1} \tilde{x}_{3}\left(w_{1}-\tilde{\psi}_{1}+\tilde{\psi}_{2}\right)}{N^{2} \tilde{\psi}_{5}=}+\kappa(t)\left(\tilde{\psi}_{4}-\tilde{\psi}_{6}\right)+\left(\lambda(t)+\tilde{u}_{3}\right)\left(\tilde{\psi}_{4}-\tilde{\psi}_{5}\right), \\
\dot{\tilde{\psi}}_{6}=\frac{\tilde{u}_{1} \beta \tilde{x}_{1} \tilde{x}_{3}\left(w_{1}-\tilde{\psi}_{1} \tilde{x}_{3}\left(w_{1}-\tilde{\psi}_{1}+\tilde{\psi}_{2}\right)\right.}{N^{2}}+w_{2}, \\
\dot{\tilde{\psi}}_{7}=\frac{\tilde{u}_{1} \beta \tilde{x}_{1} \tilde{x}_{3}\left(w_{1}-\tilde{\psi}_{1}+\tilde{\psi}_{2}\right)}{N^{2}}+w_{3} .
\end{array}\right.
$$

Note that we have obtained an analytical explicit expression for the controls $\tilde{u}_{1}(t), \tilde{u}_{2}(t)$ and $\tilde{u}_{3}(t)$,

$$
\begin{aligned}
& \tilde{u}_{1}(t)=\frac{\beta \tilde{x}_{1}(t) \tilde{x}_{3}(t)\left(\tilde{\psi}_{1}(t)-\tilde{\psi}_{2}(t)-w_{1}\right)}{N v_{1}}, \\
& \tilde{u}_{2}(t)=\frac{\tilde{x}_{1}(t)\left(\tilde{\psi}_{1}(t)-\tilde{\psi}_{7}(t)\right)}{v_{2}} \\
& \tilde{u}_{3}(t)=\frac{\tilde{x}_{4}(t)\left(\tilde{\psi}_{4}(t)-\tilde{\psi}_{5}(t)\right)}{v_{3}}
\end{aligned}
$$

but we do not have the controls in open-loop (because they depend on the state variables $\tilde{x}$ and adjoint variables $\tilde{\psi}$ ). To plot $\tilde{u}(t)$ as a function of $t$ we need to solve numerically system (5) and (10) to know the expressions for $\tilde{x}$ and $\tilde{\psi}$ and be able to obtain the controls $u_{i}, i=1,2,3$, in agreement with (11). This is done numerically in next section. For more on numerical approaches to solve optimal control problems, we refer the reader to (Alipour, 2017; Zaitri et al., 2019) and references therein.

\section{Numerical Results}

Now, our aim is to find optimal controls to limit the spread of the epidemic of COVID-19 in Italy, by reducing the number of new infections and by increasing insusceptible individuals and the percentage of those recovered, while reducing the cost during the period of three months starting from September 1, 2020. All numerical computations were performed in the numeric computing environment MATLAB R2019b using the medium order method and numerical interpolation (Shampine \& Reichelt, 1997). The rest of the preliminary conditions and real data were taken and computed from the database https://raw.githubusercontent.com/pcm-dpc/COVID-19/master/datiregioni/dpc-covid19-ita-regioni.csv. We now summarize the real data for COVID-19 pandemic in Italy, for September and October 2020.

In Tables 1 and 2, we show the real data $R(t)$ of recovered individuals from COVID-19 in Italy, September (Table 1) and October (Table 2) 2020, versus the number $R(t)$ of recovered individuals predicted by SEIR-type model (1) of (Peng et al., 2020) and the controlled model (5). We also indicate the improvement one could have done by introducing suitable controls, as explained in Section 3, and using the theory of optimal control as in Section 4. For that, we give in Tables 1 and 2 the percentage of relative error $\eta_{R}$ between real data and the one predicted by model (1); and the improvement $\mathcal{I}_{R}$ (increase of recovered individuals with respect to real data in September and up to 14-Oct-2020; and decrease of recovered from 15-Oct-2020 on, because of a drastic reduction on the number of infected and susceptible individuals) by introducing controls $u_{1}, u_{2}$ and $u_{3}$, as in (5), in an optimal control way. 
Table 1. Recovered individuals $R(t)$, Sept. 2020.

\begin{tabular}{cccccc}
\hline Day & Real & $(1)$ & $(5)$ & $\eta_{R}$ & $\mathcal{I}_{R}$ \\
\hline 01 & 207944 & 207944 & 207944 & $0 \%$ & $0 \%$ \\
05 & 209610 & 207996 & 236134 & $0.77 \%$ & $12.65 \%$ \\
10 & 211885 & 209176 & 238769 & $1.27 \%$ & $12.68 \%$ \\
15 & 214645 & 211897 & 240170 & $1.28 \%$ & $11.89 \%$ \\
20 & 218351 & 214873 & 241363 & $1.59 \%$ & $10.53 \%$ \\
25 & 222716 & 218150 & 242306 & $2.05 \%$ & $8.79 \%$ \\
30 & 227704 & 221973 & 243000 & $2.51 \%$ & $6.71 \%$ \\
\hline
\end{tabular}

Table 2. Recovered individuals $R(t)$, Oct. 2020.

\begin{tabular}{cccccc}
\hline Day & Real & $(1)$ & $(5)$ & $\eta_{R}$ & $\mathcal{I}_{R}$ \\
\hline 01 & 222832 & 224334 & 243132 & $0.67 \%$ & $9.11 \%$ \\
05 & 232681 & 226703 & 243647 & $2.56 \%$ & $4.71 \%$ \\
10 & 238525 & 232871 & 244263 & $2.37 \%$ & $2.40 \%$ \\
15 & 245964 & 241255 & 244857 & $1.91 \%$ & $0.45 \%$ \\
20 & 255005 & 252990 & 245433 & $0.79 \%$ & $3.75 \%$ \\
25 & 266203 & 269718 & 245994 & $1.32 \%$ & $7.59 \%$ \\
29 & 279282 & 288247 & 246401 & $3.21 \%$ & $11.77 \%$ \\
\hline
\end{tabular}

In Tables 3 and 4, we give real data of death individuals $D(t)$ from COVID-19 in Italy, September (Table 3) and October (Table 4) 2020, versus the number $D(t)$ of death individuals predicted by the SEIRtype model (1) of (Peng et al., 2020) and our controlled model (5). We also indicate the improvement one could have done by introducing suitable controls, as explained in Section 3, and using the theory of optimal control as in Section 4: we show the percentage of relative error $\eta_{D}$ between real data and the one predicted by model (1); and the improvement $\mathcal{I}_{D}$ (decrease of death individuals with respect to real data) by introducing controls $u_{1}, u_{2}$ and $u_{3}$, as in (5), in an optimal control way.

In Tables 5 and 6, we show the real data $Q(t)$ of quarantined individuals from COVID-19 in Italy, September (Table 5) and October (Table 6) 2020, versus the number $Q(t)$ of quarantined individuals predicted by the SEIR-type model (1) of (Peng et al., 2020) and the one predicted by our model (5). We also indicate the improvement one could have done by introducing suitable controls, as explained in Section 3, and using the theory of optimal control: we give the percentage of relative error $\eta_{Q}$ between real data and the one predicted by model (1); and the improvement $\mathcal{I}_{Q}$ (decrease of quarantined individuals with respect to real data) by introducing controls $u_{1}, u_{2}$ and $u_{3}$ in an optimal control way.

The parameters $\alpha, \beta, \gamma, \delta,\left(\kappa_{1}, \kappa_{2}, \kappa_{3}\right)$ and $\left(\lambda_{1}, \lambda_{2}, \lambda_{3}\right)$ were fitted in the least square sense.

Table 3. Death individuals $D(t)$, Sept. 2020.

\begin{tabular}{cccccc}
\hline Day & Real & $(1)$ & $(5)$ & $\eta_{D}$ & $\mathcal{I}_{D}$ \\
\hline 01 & 35491 & 35491 & 35491 & $0 \%$ & $0 \%$ \\
05 & 35541 & 35510 & 35495 & $0.08 \%$ & $0.12 \%$ \\
10 & 35597 & 35538 & 35496 & $0.16 \%$ & $0.28 \%$ \\
15 & 35645 & 35570 & 35496 & $0.21 \%$ & $0.41 \%$ \\
20 & 35724 & 35606 & 35496 & $0.33 \%$ & $0.63 \%$ \\
25 & 35818 & 35648 & 35496 & $0.47 \%$ & $0.89 \%$ \\
30 & 35918 & 35702 & 35497 & $0.60 \%$ & $1.17 \%$ \\
\hline
\end{tabular}


Table 4. Death individuals $D(t)$, Oct. 2020.

\begin{tabular}{cccccc}
\hline Day & Real & $(1)$ & $(5)$ & $\eta_{D}$ & $\mathcal{I}_{D}$ \\
\hline 01 & 35941 & 35715 & 35497 & $0 \%$ & $0 \%$ \\
05 & 36030 & 35773 & 35497 & $0.71 \%$ & $1.47 \%$ \\
10 & 36166 & 35870 & 35497 & $0.81 \%$ & $1.84 \%$ \\
15 & 36427 & 36008 & 35497 & $1.15 \%$ & $2.55 \%$ \\
20 & 36832 & 36206 & 35497 & $1.69 \%$ & $3.62 \%$ \\
25 & 37479 & 36491 & 35497 & $2.63 \%$ & $5.28 \%$ \\
29 & 38321 & 37003 & 35498 & $3.43 \%$ & $7.36 \%$ \\
\hline
\end{tabular}

Table 5. Quarantined individuals $Q(t)$, Sept. 2020.

\begin{tabular}{cccccc}
\hline Day & Real & $(1)$ & $(5)$ & $\eta_{Q}$ & $\mathcal{I}_{Q}$ \\
\hline 01 & 26754 & 26754 & 26754 & $0 \%$ & $0 \%$ \\
05 & 31194 & 29264 & 1023 & $06.18 \%$ & $96.69 \%$ \\
10 & 35708 & 31105 & 337 & $12.89 \%$ & $99.05 \%$ \\
15 & 39712 & 32183 & 228 & $18.95 \%$ & $99.42 \%$ \\
20 & 44098 & 34808 & 176 & $21.06 \%$ & $99.60 \%$ \\
25 & 47718 & 39848 & 149 & $16.49 \%$ & $99.68 \%$ \\
30 & 51263 & 48428 & 134 & $05.53 \%$ & $99.73 \%$ \\
\hline
\end{tabular}

Table 6. Quarantined individuals $Q(t)$, Oct. 2020.

\begin{tabular}{cccccc}
\hline Day & Real & $(1)$ & $(5)$ & $\eta_{Q}$ & $\mathcal{I}_{Q}$ \\
\hline 01 & 52647 & 50023 & 130 & $04.98 \%$ & $99.75 \%$ \\
05 & 58903 & 62193 & 124 & $05.58 \%$ & $99.78 \%$ \\
10 & 74829 & 83557 & 119 & $11.63 \%$ & $99.84 \%$ \\
15 & 99266 & 116035 & 116 & $16.89 \%$ & $99.88 \%$ \\
20 & 142739 & 164668 & 112 & $15.36 \%$ & $99.92 \%$ \\
25 & 222241 & 236520 & 109 & $06.42 \%$ & $99.95 \%$ \\
29 & 299191 & 317055 & 107 & $05.97 \%$ & $99.96 \%$ \\
\hline
\end{tabular}




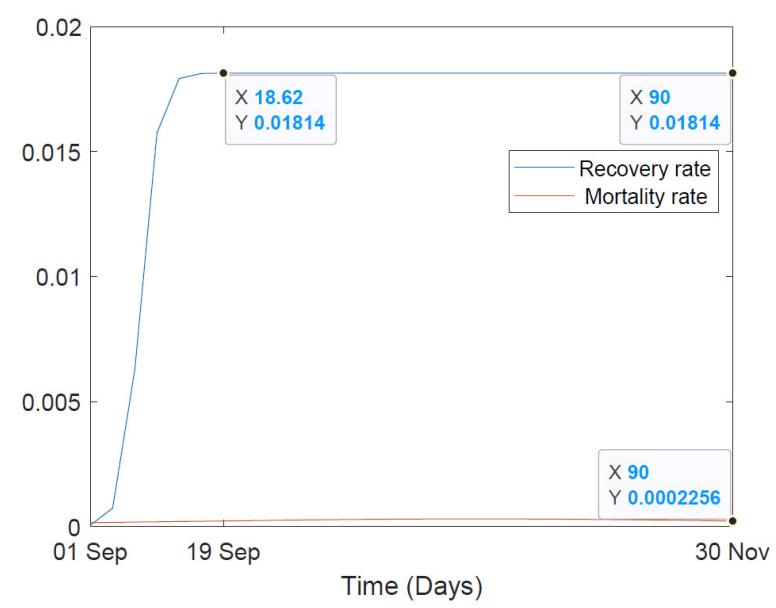

Fig. 2. The recovery and mortality rates (3) and (4) for the case of Italy (Section 5).

In Figure 2, we plot functions $\lambda(t)$ (3) and $\kappa(t)$ (4) by considering the initial guess $\alpha=0.06, \beta=1$, $\gamma=5, \delta=0.5,\left(\lambda_{1}, \lambda_{2}, \lambda_{3}\right)=(0.01,0.1,10)$ and $\left(\kappa_{1}, \kappa_{2}, \kappa_{3}\right)=(0.001,0.001,10)$, respectively.

The parameters of the generalized SEIR model (1) were computed simultaneously by the nonlinear least-squares solver (Cheynet, 2020). These parameters, during the period under study, were found as follows: $\alpha=1.1775 \times 10^{-7}, \beta=3.97, \gamma=0.0048, \delta=0.1432,\left(\lambda_{1}, \lambda_{2}, \lambda_{3}\right)=(0.0181,0.8111,6.9882)$ and $\left(\kappa_{1}, \kappa_{2}, \kappa_{3}\right)=(0.00062,0.0233,54.0351)$. For the optimal control problem of Section 4, we further fixed $w_{i}=v_{i}=1, u_{1 \min }=0.1, u_{j \min }=0, u_{i \max }=1, i=1,2,3, j=2,3$.

In Figures 3 and 4, we present plots with the numerical solutions to the nonlinear differential equations of the generalized SEIR model (1), in red color; to the nonlinear differential equations of the generalized SEIR control system (5) under optimal controls, in the sense of Section 4, in green color; and the real data of the quarantined cases, the number of recovered individuals, and the number of deaths from September 1 to October 31, 2020, in orange. The computed optimal controls for Italy from September 1 to November 30, 2020, which give rise to the green curves in the plots of Figures 3 and 4, are shown in Figure 5. The obtained simulations allow us to predict the results of the decisions taken in Italy, as well to give the best decisions for Italy, according to our generalized SEIR control system and optimal control problem.

The orange curves in Figures $3 e$ and $3 f$ and Figure 4 represent the real data on quarantine, recovered, and death cases in Italy from September 1 to October 31, 2020. The red curves simulate what happens from the beginning of September to the end of November following the generalized SEIR model (1), when the number of quarantined, recovered, and deaths increase, and reach, respectively, two million three hundred eighty-eight thousand (2388000), nine hundred six thousand three hundred (906300), and forty-five thousand seven hundred (45700) cases.

The red curves in Figures 3c, 3d and 3a simulate what happens from the beginning of September to the end of November, according with the generalized SEIR model, when the number of infected, exposed and insusceptible cases reach, respectively, one million one hundred forty-six thousand (1146000), fortyone million two hundred fifty thousand (41250000) and five hundred twenty-eight (528) cases.

The green curves in Figures 3 and 4 show what happens from September 1 to November 30, 2020, under optimal control measures, when the number of infected (Figure 3c) and recovered (Figure 3f) cases increase and reach six hundred and fifty (650) and two hundred forty-nine thousand four hundred (249400) cases, respectively, while the number of exposed (Figure 3d), insusceptible (Figure 3a), and quarantined (Figure 3e) cases reach eighteen thousand four hundred ninety (18490), sixty million one hundred eighty thousand (60180000), and one hundred twenty-eight (128) cases, respectively.

Deaths remain stable during the entire period, precisely, thirty-five thousand five hundred (35500) cases (Figure 4). 


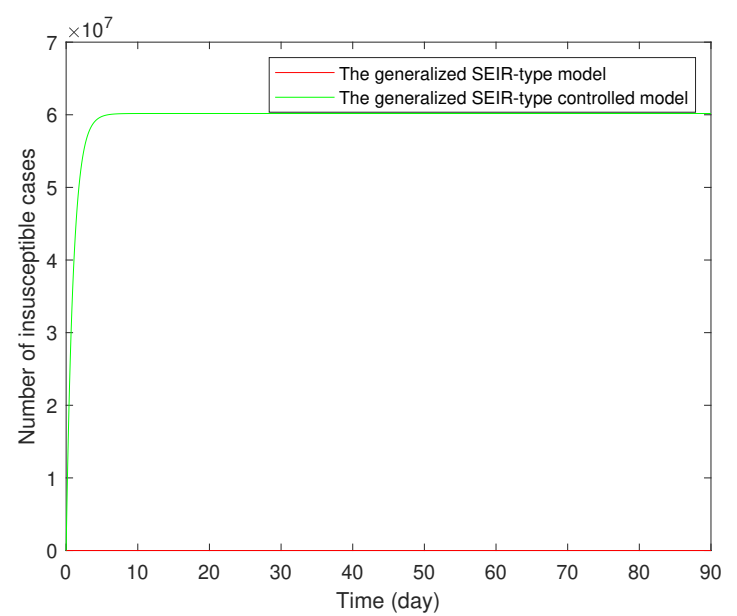

(a) $P(t)$

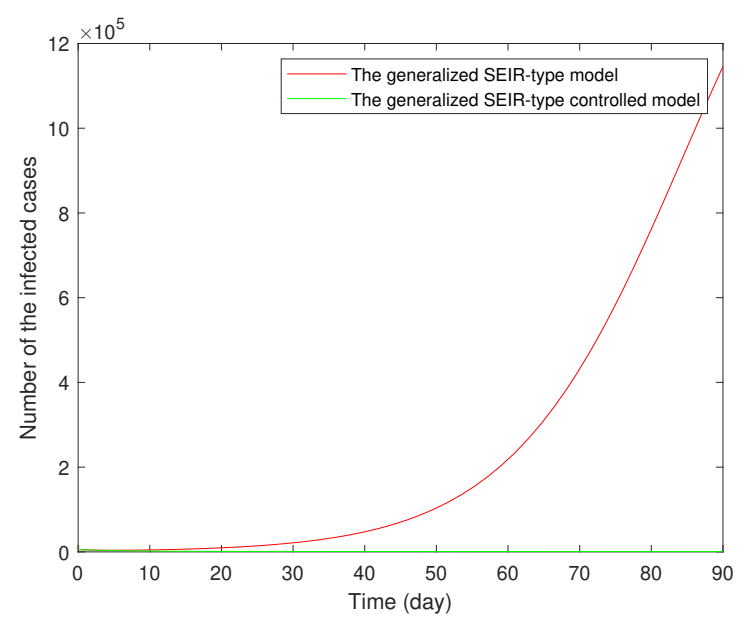

(c) $I(t)$

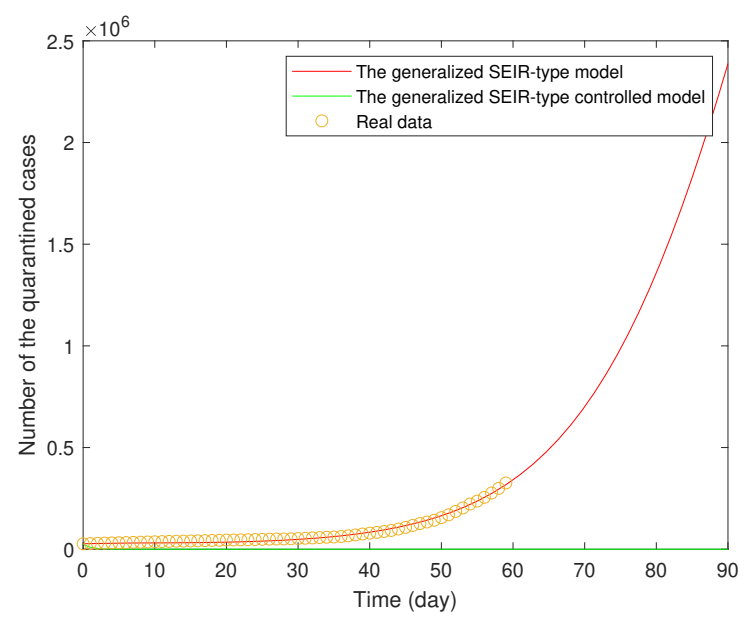

(e) $Q(t)$

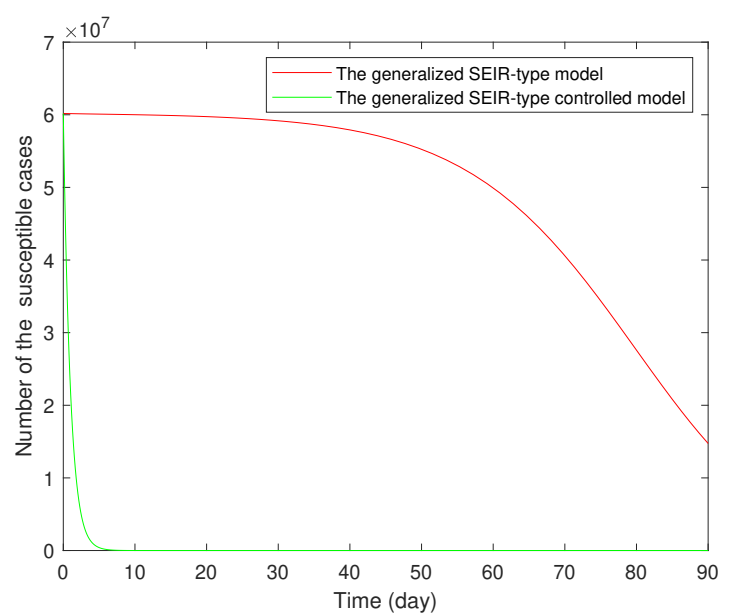

(b) $S(t)$

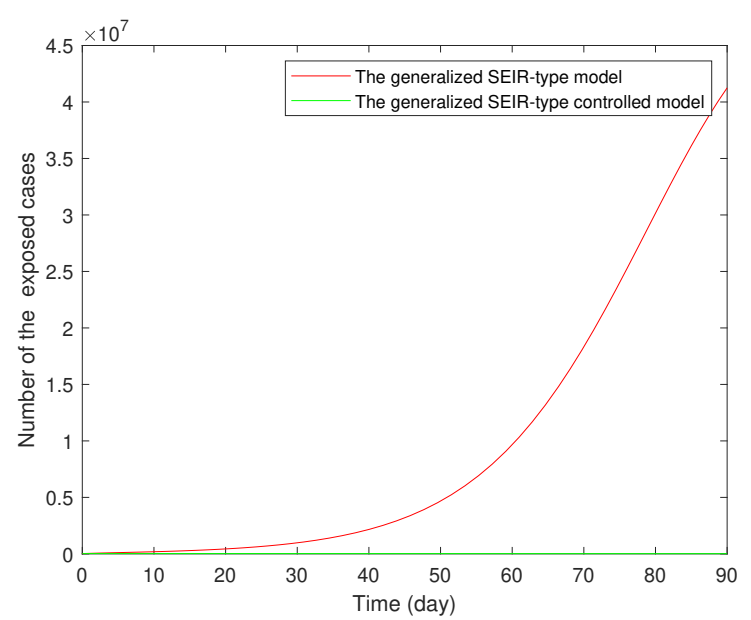

(d) $E(t)$

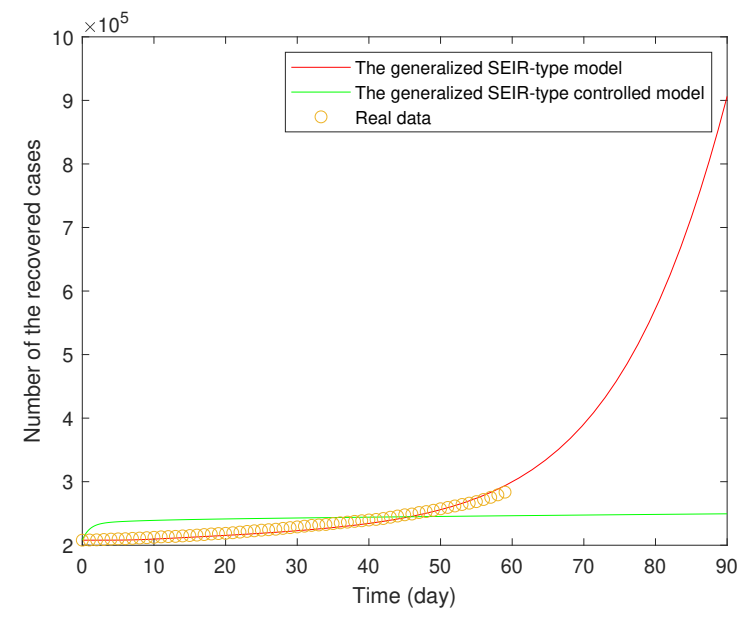

(f) $R(t)$

Fig. 3. Predictions for Italy from the generalized SEIR model (1), in red, the generalized SEIR control system (5) under optimal controls, in green, between Sept. 1 and Nov. 30, 2020, versus available real data of quarantined and recovered from Sept. 1 to Oct. 31, 2020, in orange. 


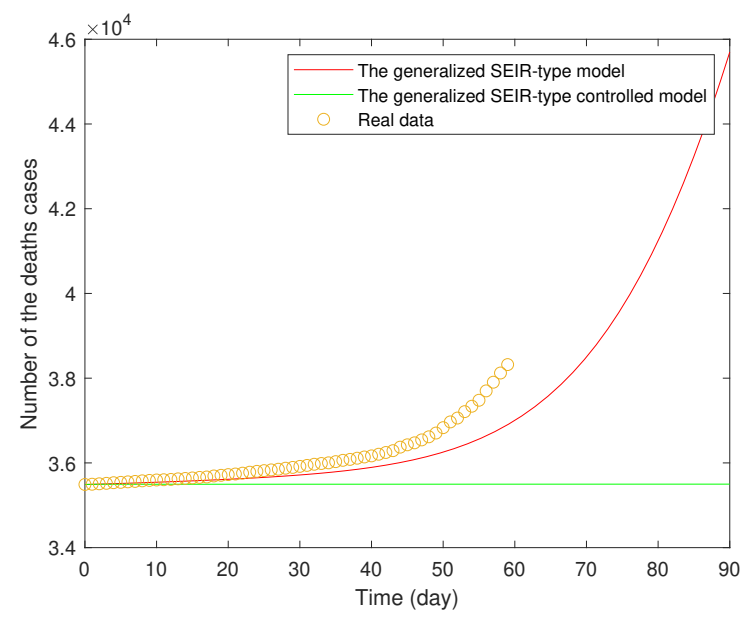

Fig. 4. Predictions for Italy from the generalized SEIR model (1), in red, the generalized SEIR control system (5) under optimal controls, in green, between Sept. 1 and Nov. 30, 2020, versus available real data of deaths from Sept. 1 to Oct. 31, 2020, in orange.

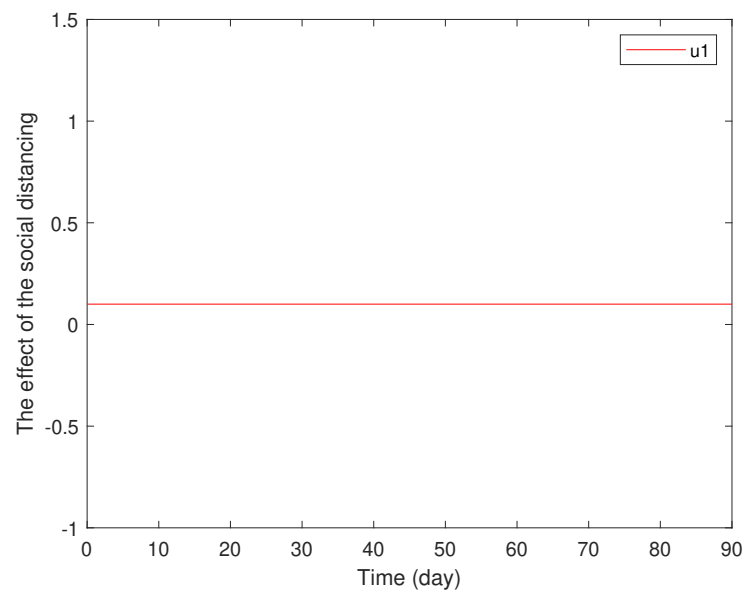

(a) $u_{1}(t)$ (social distancing)

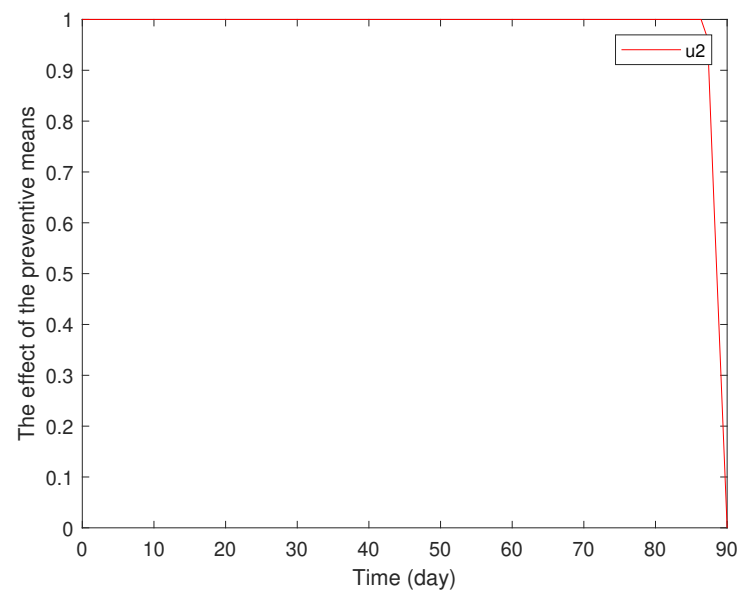

(b) $u_{2}(t)$ (preventive means)

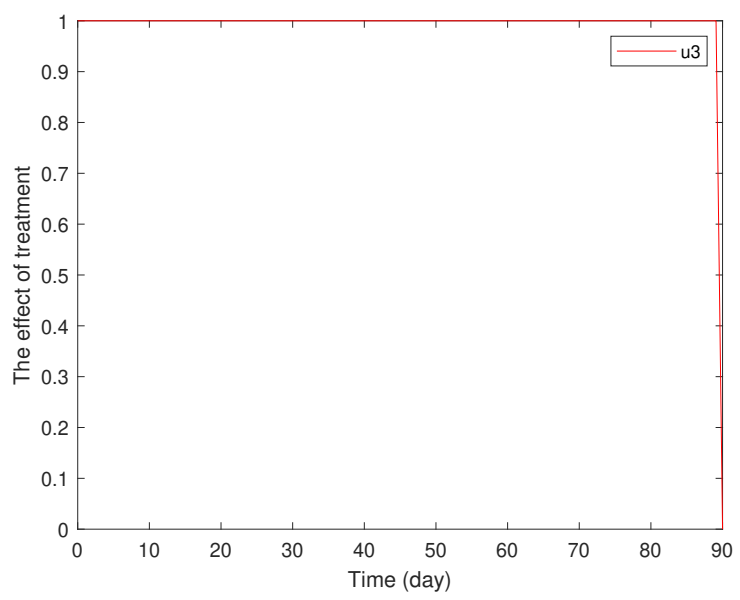

(c) $u_{3}(t)$ (treatment)

Fig. 5. The Pontryagin extremal controls of the optimal control problem of Section 4 for the case of Italy between Sept. 1 and Nov. 30, 2020. 
The curves in Figure 5 show the optimal controls that need to be implemented in order to reduce the overall burden of COVID-19 in Italy and obtain the best possible situation given by the green curves in Figures 3 and 4, which take into account the cost reduction resulting from the controls $u_{1}, u_{2}$ and $u_{3}$. The effect of social distancing is equal to the minimum value of its constraint $\left(u_{1}=0.1\right)$, see Figure 5a, and this corresponds to the application of social distancing among the entire population. The effect of preventive measures is equal to the maximum value of its constraint until September $19\left(u_{2}=1\right)$, see Figure $5 \mathrm{~b}$, then decreases gradually until it reaches zero $\left(u_{2}=0\right)$ on November 30,2020 , see Figure $5 \mathrm{c}$. The effect of treatment takes the maximum value of its constraint until November 29, $2020\left(u_{3}=1\right)$, then decreases to zero on November 30, $2020\left(u_{3}=0\right)$, meaning a decrease in the pressure on the health sector. Note that by taking preventive measures $\left(u_{1}, u_{2}, u_{3}\right)$, we limit the spread of COVID-19 and we have better results. This means that, with the help of optimal control theory, what happened in Italy would have been less dramatic.

\section{Conclusion}

Recent results have shown how the theory of optimal control is an important tool to combat COVID-19 in a community: in (Silva et al., 2021) for a controlled sanitary deconfinement in Portugal; in (Zamir et al., 2021) from a more theoretical point of view; here for the case of Italy. We proposed a simple SEIR-type control system, showing its effectiveness with respect to real data from Italy in the period from September 1 to November 30, 2020. While the real data is consistent with the generalized SEIR model (1), because the goal of this model is to describe well the COVID-19 reality, our new SEIR control system (5) simulates what would happen if we took into account the values of the three control functions, as described in Section 3. In agreement, the situations obtained with controls are better than the situations obtained without controls. More precisely, by considering the proposed controls, we show how optimal control theory could have drastically diminish the burden of COVID-19 in the period under study while taking into account the resulting cost reduction. In concrete, if it would have been possible to implement optimally, in the sense of optimal control theory and Pontryagin's optimality conditions, the control measures of social distancing as in Figure 5a, preventive means as in Figure 5b, and treatment as in Figure 5c, then it would have been possible to decrease significantly the number of deaths (cf. Figure 4 and Tables 3 and 4, which account a decrease of 7.36\% of deaths in Italy by the end of October 2020 under optimal control) with much less quarantined individuals (see Figure $3 e$ and Tables 5 and 6 , which account a decrease of $99.96 \%$ of quarantined individuals in Italy by the end of October 2020 under optimal control theory). Thus, one can say that the approach proposed by the theory of optimal control is very effective, simultaneously from health and economical points of view, being far from trivial. Note that by following Pontryagin's minimum priciple one obtains an increase on the number of recovered individuals in a first period, up to 14-Oct-2020, and, after this date, a decrease on the number of recovered (cf. Figure $3 \mathrm{f}$ and Tables 1 and 2), caused by the drastic reduction on the number of susceptible and infected (see Figures $3 b$ and $3 c$, respectively).

While our aim here was to study the effect of controls, guided by application of the Pontryagin minimum principle and showing how they can help to decrease the spread of COVID-19, other aspects remain open for further research. In particular, it remains open the theoretical study of the stability of the models. In this direction, the recent results of (Boukhouima et al., 2021; Ndaïrou \& Torres, 2021) may be useful.

\section{ACKNOWLEDGMENTS}

This research is part of first author's Ph.D. project. Zaitri is grateful to the financial support from the Ministry of Higher Education and Scientific Research of Algeria; Torres acknowledges the financial support from CIDMA through project UIDB/04106/2020.

\section{References}

Alipour, M. (2017). Numerical study on multi-order multi-dimensional fractional optimal control problem in general form, Kuwait J. Sci. 44(3), 9-19. 
Bagal, D. K., Rath, A., Barua, A., \& Patnaik, D. (2020). Estimating the parameters of susceptibleinfected-recovered model of COVID-19 cases in India during lockdown periods, Chaos Solitons Fractals 140, Art. 110154, 12 pp.

Barril, C., Calsina, A., Cuadrado, S., \& Ripoll, J. (2021). On the basic reproduction number in continuously structured populations, Math. Methods Appl. Sci. 44(1), 799-812.

Boukhouima, A., Lotfi, E. M., Mahrouf, M., Rosa, S., Torres, D. F. M., \& Yousfi, N. (2021). Stability analysis and optimal control of a fractional HIV-AIDS epidemic model with memory and general incidence rate, Eur. Phys. J. Plus 136, Art. 103, 20 pp.

Cesari, L. (1983). Optimization-theory and applications, Applications of Mathematics (New York), 17, Springer-Verlag, New York.

Cheynet, E. (2020). Generalized SEIR Epidemic Model (fitting and computation), https:// github.com/ECheynet/SEIR/tree/v4.8.7

Kermack, W. O. \& McKendrick, A. G. (1927). A contribution to the mathematical theory of epidemics, Proc. R. Soc. Lond., Ser. A 115, 700-721.

Lauer, S. A., Grantz, K. H., Bi, Q., Jones, F. K., Zheng, Q., Meredith, H. R., Azman, A. S., Reich, N. G. \& Lessler, J. (2020). The incubation period of coronavirus disease 2019 (COVID-19) from publicly reported confirmed cases: Estimation and application, Annals of Internal Medicine 172(9), 577-583.

Lemos-Paião, A. P., Silva, C. J., \& Torres, D. F. M. (2020). A new compartmental epidemiological model for COVID-19 with a case study of Portugal, Ecological Complexity 44, Art. 100885, 8 pp.

Q. Lin, S. Zhao, D. Gao, Y. Lou, S. Yang, S. S. Musa, Wang, M. H., Cai, Y., Wang, W., Yang, L. \& He, D. (2020). A conceptual model for the coronavirus disease 2019 (COVID-19) outbreak in Wuhan, China with individual reaction and governmental action, International Journal of Infectious Diseases 93, 211-216.

Lu, R., et al. (2020). Genomic characterisation and epidemiology of 2019 novel coronavirus: Implications for virus origins and receptor binding, The Lancet 395(10224), 565-574.

Moussaoui, A. \& Auger, P. (2020). Prediction of confinement effects on the number of Covid-19 outbreak in Algeria, Math. Model. Nat. Phenom. 15, Paper No. 37, 14 pp.

Ndaïrou, F., Area, I., Nieto, J. J., Silva, C. J. \& Torres, D. F. M. (2021). Fractional model of COVID-19 applied to Galicia, Spain and Portugal, Chaos Solitons Fractals 144, Art. 110652, 7 pp.

Ndaïrou, F. \& Torres, D. F. M. (2021). Mathematical analysis of a fractional COVID-19 model applied to Wuhan, Spain and Portugal, Axioms 10(3), Art. 135, 13 pp.

Peng, L., Yang, W., Zhang, D., Zhuge, C. \& Hong, L. (2020). Epidemic analysis of COVID-19 in China by dynamical modeling, preprint, arXiv:2002.06563v2.

Pontryagin, L. S., Boltyanskii, V. G., Gamkrelidze, R. V. \& Mishchenko, E. F. (1962). The mathematical theory of optimal processes, Translated from the Russian by K. N. Trirogoff; edited by L. W. Neustadt, Interscience Publishers John Wiley \& Sons, Inc. New York.

Shampine, L. F. \& Reichelt, M. W. (1997). The MATLAB ODE suite, SIAM J. Sci. Comput. 18(1), $1-22$.

Silva, C. J., et al. (2021). Optimal control of the COVID-19 pandemic: controlled sanitary deconfinement in Portugal, Scientific Reports 11, Art. 3451, 15 pp. 
van den Driessche, P. \& Watmough, J. (2002). Reproduction numbers and sub-threshold endemic equilibria for compartmental models of disease transmission, Math. Biosci. 180, 29-48.

Zaitri, M. A., Bibi, M. O. \& Bentobache, M. (2019). A hybrid direction algorithm for solving optimal control problems, Cogent Math. Stat. 6(1), Art. 1612614, 12 pp.

Zamir, M., Abdeljawad, T., Nadeem, F., Wahid, A. \& Yousef, A. (2021). An optimal control analysis of a COVID-19 model, Alexandria Engineering Journal 60(3), 2875-2884.

Zhao, Z., Li, X., Liu, F., Zhu, G., Ma, C. \& Wang, L. (2020). Prediction of the COVID-19 spread in African countries and implications for prevention and control: A case study in South Africa, Egypt, Algeria, Nigeria, Senegal and Kenya, Science of the Total Environment 729, Art. 138959, 10 pp.

Zine, H., Boukhouima, A., Lotfi, E. M., Mahrouf, M., Torres, D. F. M. \& Yousfi, N. (2020). A stochastic time-delayed model for the effectiveness of Moroccan COVID-19 deconfinement strategy, Math. Model. Nat. Phenom. 15, Art. 50, 14 pp.

$\begin{array}{ll}\text { Submitted } & : 28 / 04 / 2021 \\ \text { Revised } & : 30 / 06 / 2021 \\ \text { Accepted } & : 25 / 07 / 2021 \\ \text { DOI } & : 10.48129 / \text { kjs.splcov.13961 }\end{array}$

